\title{
Extracting Behavioural Patterns from a Negotiation Game
}

\author{
Marco Gomes, Tiago Oliveira, Davide Carneiro, Paulo Novais and José Neves \\ Department of Informatics, University of Minho \\ pg18373@alunos.uminho.pt, \\ \{dcarneiro, toliveira,pjon, jneves\}@di.uminho.pt
}

\begin{abstract}
The work presented focuses not only on the behavioural patterns that influence the outcome of a negotiation, but also on the discovery of ways to predict the type of conflict used in the process and the stress levels of the actors. After setting up an experimental intelligent environment provided with sensors to capture behavioural and contextual information, a set of relevant data was collected and analysed, with the underlying objective of using the behavioural patterns (obtained by statistical/probabilistic methods) as a basis to design and present plans and suggestions to the associated participants. In sooth, these proposals may influence in a positive way the course and outcome of a negotiation task in many aspects. This work highlights the importance of knowledge in negotiation, as in other social forms of interaction, providing also some new insights for informed decision support in situations in which uncertainty and conflict may be present.
\end{abstract}

Keywords: Intelligent Environments, Online Dispute Resolution, Negotiation, ContextAware

\section{Introduction}

Negotiation [10] is a collaborative and informal process by means of which parties communicate and, without external influence, try to reach an outcome that may satisfy both. This process is widely used in the most different fields, including legal proceedings, divorces, parental disputes or even hostage affairs. It may be also used as a mechanism to solve disputes without recurring to the traditional judicial process, i.e., litigation in courts. Undoubtedly, it stands for a highly interdependent process in which each party continuously incorporates information from the other party(ies) to devise answers that might lead to the resolution of the conflict at hand, in the quest for understanding the process through which conflicts are settled. In this particular case, negotiation is incorporated in Online Dispute Resolution (ODR) software and used in a technological context, either supported by technology or under a virtual computational environment. Indeed, being able to capture behaviour patterns performed within a negotiation is very relevant to drive the process. Therefore, modelling this human activity must take into consideration the dynamic, adaptive, and interactive setting of the virtual computational environment in which the negotiation (related to ODR) occurs. 
To acquire this kind of contextual and behavioural information, a set of models was developed. Indeed, the information from physical sensors, named low-level context, may be meaningless, trivial, vulnerable to small changes, or uncertain [12]. A way to mitigate this problem is the extraction of high level context information from raw sensor values [1] in order to attain descriptions of human behaviour that may be relevant to a negotiation. Such specifications include the negotiation style or strategy and the stress state of the parties (when facing a negotiation process). To assess this kind of information the models introduced in [4] were used to classify the negotiation/conflict resolution styles along with a multimodal approach to identify and classify a party's stress progress during a negotiation in a contextually rich and dynamic environment [8].

This work illustrates the process used to extract behavioural patterns from data gathered in a negotiation, performed within an intelligent environment, using game theory. In particular, it focuses on the analysis of behavioural data related to the estimation of stress levels and negotiation/conflict approaches of the actors. The intention is to enrich the knowledge about user states in negotiation processes for the further development of a reasoning system that will generate proposals that may show the way to successful negotiation outcomes.

\section{Systematic Behavioral Analysis}

The behaviour of a living system includes all the activities that the system would not fulfil if it were not living, with living being interpreted either in the classical sense or referring, for example, to a virtual computational machine.

These activities are always the response of the system to some stimuli, or lack of them. Stimuli are said to be internal, when they are originated inside the body of the system (e.g. thought, pain, change of state). They are said to be external when originated outside of the body of the system and perceived by some receptor cell (e.g. change in the temperature, visual change in the environment, reception of a given message).

The system may be conscious or unconscious of the perceived stimuli. Despite this, the system may respond. This response is said to be voluntary when the system undergoes some reasoning process before acting or involuntary when the system reacts in pre-determined ways, without reasoning about it.

Nothing characterizes an individual better than his/her behaviour. Knowing how an individual reacts to stimuli allows one to foresee their future states. On the other hand, controlling stimuli may allow one to control any individual. This is the way to behaviourism. Therefore, in psychology, the behaviour is influenced an studied in order to address behavioural issues of an individual, ranging from psychological disorders to other matters, such as smoking habits or eating disorders, just to name a few.

In this work, the interest is on knowing how a given party acts in response to specific scenarios (e.g. how does a party behave when under stress or during a negotiation). Introducing the figure of a mediator with access to this information, he/she will be able to make better decisions. As an example, if a mediator knows that a given party generally assumes a highly competitive style during a negotiation, he may try to show that party that such a style might be an obstacle for a successful outcome. 
The approach followed focuses on acquiring context information that allows to characterize the behaviour of the human users of the negotiation tool. Moreover, it does it in an absolutely transparent and non-invasive way, i.e., rather than relying on traditional self-reporting mechanisms such as questionnaires in order to infer behaviours, it analyses the actions of the parties, in real-time.

In order to implement such processes, procedures used in social science were analysed. In particular, an algorithm defined by [6] was followed. It provides a complete description of the procedures and principles required to identify the behaviour sources and to perceive not only the relationship between sources and behaviours but how to adjust them in order to influence the doings as preferred.

According to [6], all behavioral research should include:

1. At least one participant;

2. At least one behaviour (which stands for the dependent variable);

3. At least one setting or environment;

4. A system for measuring the behaviour and ongoing visual analysis of data;

5. At least one treatment or intervention condition;

6. Manipulations of the independent variable so that its effects on the dependent one may be quantitatively or qualitatively analysed; and

7. An intervention that will benefit the participant in some way.

The conditions for performing the experiment included the following: ten individuals participated in the study; the behaviour under consideration was the individual's conflict handling style; the environment of the experiment was the Intelligent Systems Lab, at the University of Minho; a system for measuring the behaviour in real-time was developed (it allows one to grasp the evolution of the conflict handling in real-time); the intervention condition denotes that one or more parties evidence negative conflict handling styles (e.g. competitive, avoiding) and should be guided in a proper way, i.e., by letting them know the potential consequences of particular decisions in conflict styles. It is expected that the participants will benefit from this kind of intervention, in the sense that, by improving their negotiation behaviour, the process is more likely to succeed.

\section{An Intelligent Environment to extract Behavioral Information}

The variety of user types that can be involved in each Intelligent Environment (IE), and the multitude of potential objectives of each particular environment demands an exhaustive analysis of all components to be included. Therefore, an intelligent environment with diverse devices and functionalities was built, aiming to provide the user's context and state of information to the applications being used. In this case, the developed prototype was in the area of Online Dispute Resolution (ODR).

\subsection{Assessing the level of stress}

In order to assess the level of stress of the parties, a group of devices is considered in the user-area network (Fig. 1). The main requirement when selecting the devices is 


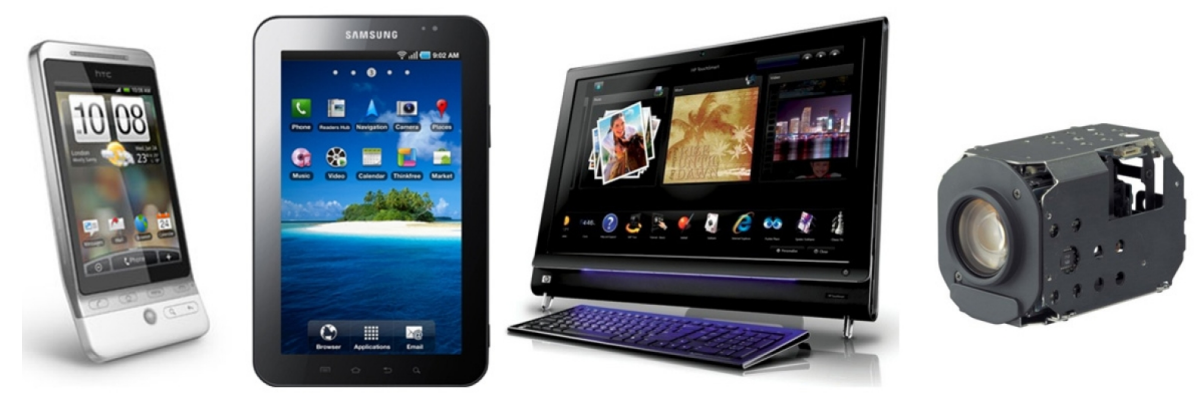

Fig. 1. Devices used to implement the described functionalities.

that they have to provide as much information about the user environment as possible. Moreover, the user has to feel comfortable with them. In that sense, the selected devices are more or less common nowadays; so they do not represent a drawback for the user. Table 1 briefly describes each device and the main characteristics of interest.

Table 1. Brief description of the functionalities of the devices that constitute the environment.

\begin{tabular}{|l||l|l|}
\hline Device & Brief description & Main features \\
\hline HP Touchsmart & All-in-one PC & touchscreen, web cam, large screen \\
\hline Samsung Galaxy Tab & Tablet PC & $\begin{array}{l}\text { touchscreen, web cam, accelerometer, } \\
\text { relatively large screen, mobile, Android } \\
\text { OS }\end{array}$ \\
\hline HTC PDAs & $\begin{array}{l}\text { touchscreen, camera, accelerometer, } \\
\text { mobile, Android OS }\end{array}$ \\
\hline Sony FCB-EX780BP & $\begin{array}{l}\text { 25x Super HAD PAL Color } \\
\text { Block Camera with Exter- } \\
\text { nal Sync Optical Zoom, Image stabilizer, } \\
\text { Day/Night Mode, Privacy Zone Mask- } \\
\text { ing }\end{array}$ \\
\hline
\end{tabular}

The focus is on devices capable of acquiring data about the behaviour of the users that can be related to stress. The following sources of information (from now on designated sensors), acquired from the respective devices, are considered:

- Touch pattern - the touch pattern represents the way in which a user touches the device and represents a variation of intensity over a period of time. This information is acquired from touchscreens with support for touch intensity.

- Touch accuracy - a comparison between touches in active controls versus touches in passive areas (e.g. without controls, empty areas) in which there is no sense in touching. This information is acquired from touchscreens.

- Touch intensity - the intensity of the touch represents the amount of force that the user is putting into the touch. It is analyzed in terms of the maximum, minimum and mean intensity of each touch. This information is acquired from touchscreens. 
- Touch duration - this represents the time span between the beginning and the end of the touch event. This data is acquired from devices with touchscreens.

- Amount of movement - the amount of movement represents how and how much the user is moving inside the environment. An estimation of the amount of movement from the video camera is built. The image processing stack uses the principles established by [5] and uses image difference techniques to calculate the amount of movement between two consecutive frames [7].

- Acceleration - the acceleration is measured from accelerometers in mobile devices. It is useful for building an estimation of how much the user is moving and how he is doing it (e.g. is the user having sudden movements?). Moreover, information from the accelerometer is used to support the estimation of the intensity of touch.

The stress models used in this work were built in a previous experiment. The collection of the data was organized in two phases. In a first phase, participants were required to perform specific tasks through the interaction with the devices in a stress-free environment. In a second phase, they performed similar tasks subject to stressors such as the vibration of the devices, loud and annoying sounds, unexpected behaviors of the devices, among others.

The empirical data gathered in both phases about the participant's interaction patterns and physical response was synchronized and transformed/normalized to allow its joint analysis. The participants of the proposed experiment were volunteer students and professors from our institution. 19 male and female individuals participated in the experiment aged between 20 and 57. All these individuals are familiar with the technological devices used thus the interaction with them was not an obstacle.

The data gathered was analyzed in order to determine statistically significant differences between phase 1 and phase 2 of the data collection. Measures of central tendency and variability were calculated for all variables of interest. The Mann-WhitneyWilcoxon Statistical test was used to test whether there are actual differences in the distributions of the data. A 0.05 level of significance was considered. The data analysis was performed using Wolfram Mathematica ${ }^{\circledR}$, Version 8.0.

Based on this analysis of the data we were able to determine which parameters, for each individual, were effectively affected by stress. Using this knowledge, we developed personalized models for stress estimation in real time. Moreover, a more generic model was also developed taking into consideration the data of all the participants. This generic model can be applied in the cases in which a personalized one is not available. The whole process, including the dataset and the results, is further described in [2].

\subsection{Assessing the Conflict Handling Style from the Utility of the Proposals}

The style of dealing with a conflict that each one has must be seen as having a preponderant role in the outcome of a conflict resolution process, especially on those in which parties interact directly (e.g. negotiation, mediation). Ultimately, it is acceptable to state that the outcome will largely depend on the conflict resolution style of each party and on the interaction of the styles of the parties.

Different approaches can be followed to formalize the way that we respond to conflicts. A well-known definition was presented by Kenneth Thomas and Ralph Kilmann, 
which encoded the way that we react under a conflict into five different modes. To define these modes, they take into consideration the individual's assertiveness, which denotes how much a party tries to satisfy his own interests, and the cooperativeness, which denotes to which extent the party is willing to satisfy the other's interests. The five different conflict resolution styles defined are as follows:

- Competing - A party that shows this uncooperative style aims at maximizing his own gain, with a consequent minimization of the others. Usually, a competing individual will use his ability to argue, his rank, his social status or whatever advantageous position that he can have to show dominance over the other party. This is thus a power-oriented style;

- Accommodating - An accommodating party will show a behaviour that can be classified as the opposite of a competing one in the sense that he will be cooperative. It may happen that an accommodating party will even neglect his own gain, thus maximizing the one of the others, in order to achieve a solution. Thus, it may be said that there is an element of self-sacrifice. Generally, such a party will tend to show generosity or charity, will be understanding and will easily obey other's orders or desires even if they represent a drawback;

- Avoiding - An individual that shows an avoiding behaviour is most likely not dealing with the conflict as he usually satisfies neither his own interests nor those of the other party. Common behaviours in this conflict style include diplomatically sidestepping or postponing some issue or even withdrawing from threatening or unpleasant situations;

- Collaborating - On the opposite side of avoiding is the collaborative behaviour. This is a cooperative style in which the party shows the willingness to work with the other party in order to find solutions that can be interesting for both. This implies that the party is interested in finding what the fears and desires of the other are and might even try to explore a disagreement in order to learn from other's insights;

- Compromising - A compromising party will generally try to find a fast and satisfactory solution that can be interesting for both parties. This conflict style can be seen as an intermediary one between the competing and the accommodating. A compromising party is generally willing to split the differences between two positions, to exchange some concessions or to seek middle-ground solutions.

The potential relation between the personal conflict style and the utility of the proposals for the resolution of the conflict has been explored in previous work [3]. The utility quantifies how good a given outcome is for a party. In that sense, it is acceptable to argue that a competing party will generally propose solutions that maximize its own utility in expense of that of the other party, while for example a compromising party will most likely search for solutions in an intermediary region. Essentially, we were able to classify the personal conflict style of a party by constantly analysing the utility of the proposals created. The relation between the utility of the proposals and the conflict style is depicted in Figure 2 and further detailed in [3].

\subsection{Experiments and Datasets}

The negotiation game simulates a business situation involving a manufacturer and a reseller, in which each party has to achieve a desired result in the negotiation or go 


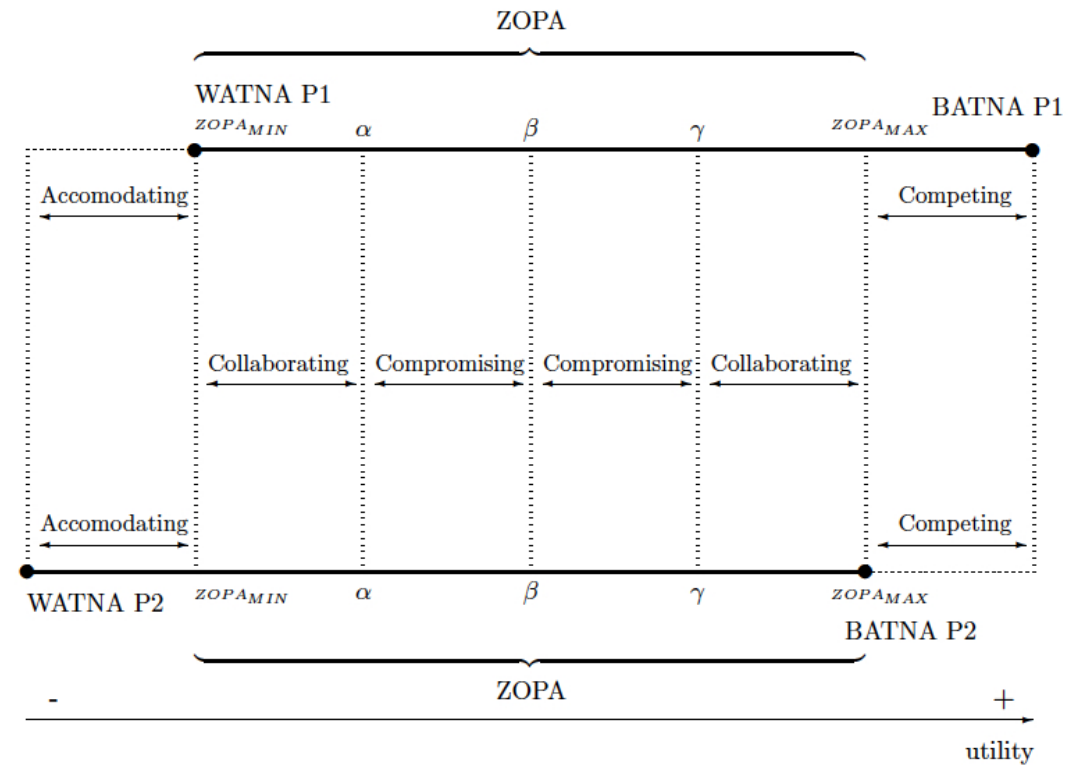

Fig. 2. The relationship between the utility of a proposal and the personal conflict handling style

bankrupt. The desired result was a win/win situation for both parties. The game starts with a random draw of roles (i.e. manufacturer or reseller) for each party. The instructions to win the game were to negotiate a successful deal and make sure that any party did not go bankrupt.

To capture behavioural and contextual information, a test environment was set up in our lab. In this environment, the users were isolated from external stimuli and had to play a negotiation game that implied interaction with portable devices. It was developed a sensor-based application for the Android Operating System (Figure 3). The choice of a mobile platform relied on the use of computer based systems that had the capability of sense, interaction, store and manage sensor-based data with portability and platform-independence requirements, like smartphones and tablet computers. The developed application covers the functional needs to perform within an IE. Thus, at each round, the application collects the sensor information (using the classes provided by the Android API) and negotiation information (e.g., the proposal, the text messages) in terms of a temporal window. During the experiments, the information about the user's context was provided by a monitoring infrastructure, which is customized to perform movement detection from a camera located in front of the user and collect and treat the information that comes from the portable devices. The group of sensors used and their associated features included touch intensity (higher levels of touch intensity are associated with increased levels of stress); and touch accuracy (this is a measure of the amount 

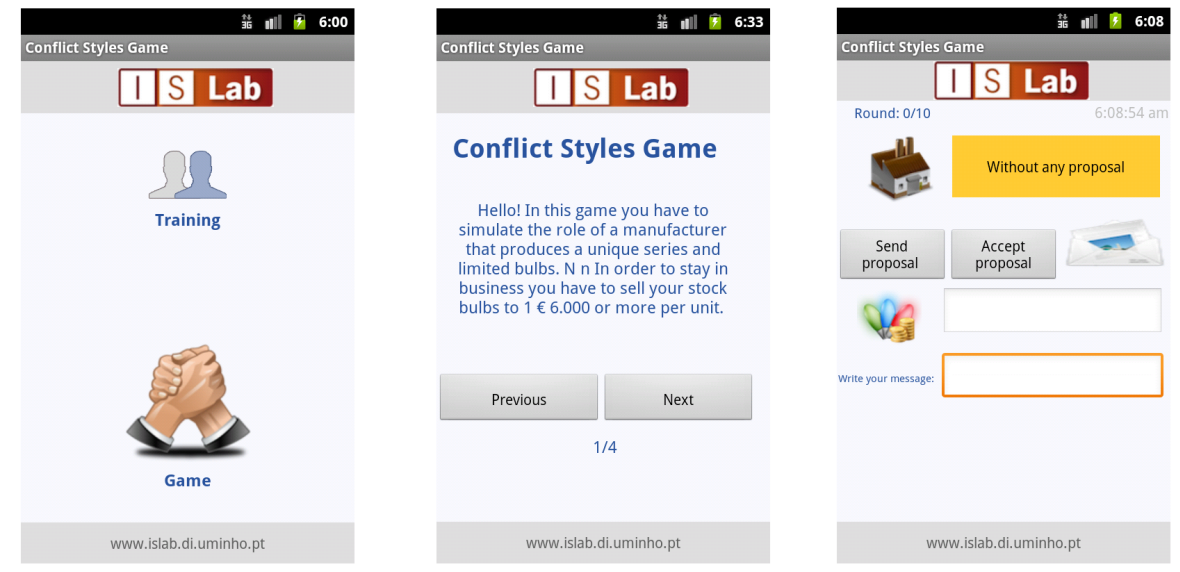

Fig. 3. Some Android-based application's graphic interfaces.

of touches in active controls versus touches in active areas). The resulting datasets are summarized in Table 2.

Table 2. Summary of the data generated during the tests. The size of the datasets comprises all the data generated.

\begin{tabular}{|l|l|l|}
\hline data & brief description & size of dataset \\
\hline Acceleration & $\begin{array}{l}\text { Data concerning the acceleration felt on the handheld device } \\
\text { while playing the game }\end{array}$ & 33366 \\
\hline Movement & $\begin{array}{l}\text { A dataset containing information about the amount of move- } \\
\text { ment during the tests }\end{array}$ & 9137 \\
\hline Touches & This dataset contains information about the touches & 590 \\
\hline Proposals & Data concerning the proposals made by the parties in all rounds & 59 \\
\hline
\end{tabular}

\subsection{The Resulting Behavioural Patterns}

Looking over the data it may be concluded that most of the time the parties are using a competitive style, either in stress or calm phases. The evaluation of the progress of the conflict styles during the negotiation process was centred on the average slope of its numeric values. It was concluded that in a stressful state the parties tend to vary slowly their way of dealing with the conflict than when they are calmer. Regarding at proposal values made by the parties during the negotiation, similar conclusions may be made. At the training phase both parties change more quickly their conflict styles than 
during the stressful phase. In the same way, manufacturers present a more dynamic proposal evolution than the resellers. It may be concluded that in a stressful situation it is more likely that the parties propose more uncooperative offers than others in a different situation. This may be explained as a consequence of acting too quickly or relying too much on coercion. When parties are under pressure they may make strategic mistakes or unwanted concessions. It may also lead to bad agreements. These are the natural assumptions that one may draw from these results. Acting too quickly may be a response to external and internal stressors. Indeed, considering the duration of the rounds, one may state that a high percentage of the negotiation rounds performed were shorter under a stressful environment than a stress-free one. However, only a small amount of these cases were statistically significant.

Looking at the statistical data one notices that $80 \%$ of the participants used a competitive conflict style in the early moves, 55\% improve their styles (shifting towards more cooperative solutions), $35 \%$ do not change their competitive style until the end, and $10 \%$ become even more competitive. It is stated that competitors often use power as the primary tool for handling conflict, and work to prove the importance of one side of the argument in order to win. This may be one of the explanations. Otherwise, they are usually more concerned with winning the game than finding the best solution. Taking into consideration the game pre-conditions, the second hypothesis seems more appropriate, but one may not extrapolate the given results. Additional insights are needed in order to have a better and a more broad explanation.

\section{A Probabilistic Model for Negotiation Posture Recognition}

Probabilistic models are considered to be one of the best ways to deal with uncertainty in a given domain [11]. When there is a need to predict something based upon relationships that are not yet transparent among variables, probabilistic models offer a comprehensive way to work out the problem. As such, the application of these models to the data collected from the experiment was considered.

\subsection{The Probabilistic Network Formalism}

To discover possible hidden relationships among the data, we look at Bayesian Networks (BNs) using machine learning techniques. BNs are graphical representations of statistical dependences and independences among variables [9]. The reasons for choosing this representation are related with the fact that a $\mathrm{BN}$ provides a network structure and a probability distribution that are easily interpreted by humans and machines, as well as a comprehensive way of dealing with uncertainty. In fact, there are a number of programs that enable a user to manipulate $\mathrm{BN}$ parameters and selectively analyse the impact of these changes.

A BN is an acyclic directed graph $G=(V(G), A(G)$ with a set of vertices $V(G)=$ $\left\{V_{1}, \ldots, V n\right\}$, where each vertex $V_{i} \in V(G)$ represents a discrete stochastic variable, and a set of $\operatorname{arcs} A(G) \subseteq V(G) \times V(G)$, where each $\operatorname{arc}\left(V_{i}, V_{j}\right) \in A(G)$ represents statistical dependence. A BN defines a joint probability distribution $\operatorname{Pr}$ that may be factorized in 
the following way:

$$
\operatorname{Pr}\left(V_{i}, \ldots, V_{n}\right)=\prod_{i=1}^{n} \operatorname{Pr}\left(V_{i} \mid \pi\left(V_{i}\right)\right)
$$

where $\pi\left(V_{i}\right)$ is associated with the set of variables that denote the parents of $V_{i}$.

\subsection{The Model for Negotiation Style Recognition}

Before feeding the data to the learning algorithms, some pre-processing was needed, namely the conversion of the variables round number, proposal value and round duration to nominal variables. The dataset included 103 instances and the set of variables used to acquire knowledge about the model were:

- ExperienceType: if the experience occurred under stress conditions or under training conditions;

- Round: the round interval to which the instance belongs;

- Part: if the role played in the instance was a manufacturer or a reseller;

- ProposalValue: the value in euros of the proposal;

- ConflictStyle: : the conflict style detected in the negotiation; and

- RoundDuration: the estimated duration of the round in terms of time intervals.

There are essentially two ways of learning the structure of BNs: score-based search and constraint-based search [9]. Score-based algorithms search for a BN structure that better fits the data by starting with an initial network and then traversing the search space of structures, removing, adding or reversing arcs in each step. On the other hand, constraint-based algorithms carry out a conditional (in)dependence analysis on the data. Based on this analysis, an undirected graph is generated to be interpreted as a Markov network. Given the reduced number of available instances, the method used was scorebased search since it appears to work better in these cases. The algorithms used to learn the topology of the network were the hill-climbing and the tabu algorithms which are available in the bnlearn package for R. When retrieving the score (measure of fitness of the network to the data) for each network, the one learned by hill-climbing was the one with the highest, and so it was the one used to learn the probability distribution of the parameters. The retrieved network is represented in Figure 4. When one observes the $\mathrm{BN}$ of Figure 4 it is visible that it is rather sparse, probably due to the small amount of data used to construct it. None of the algorithms used managed to establish a dependence relationship between the ConflictStyle and the other variables under study, which does not fit the desired outcome of the process. However, it is noticeable that the competing style is the most frequent. Some other relationships were detected, namely between Part and ProposalValue. When the manufacturer part is taken, the value of the proposals tends to be at lower intervals than when the reseller part is the one chosen. Again, the algorithms did not establish a connection between these two vertices and the other variables. By observing the remaining network, one detects some diverging arcs between Round, ExperienceType and RoundDuration which means that instantiating ExperienceType blocks the flow of probabilistic information, i.e., the influence from the Round interval to the RoundDuration (and vice versa). This means that if the ExperienceType is not instantiated, instantiating the Round may change the marginal 


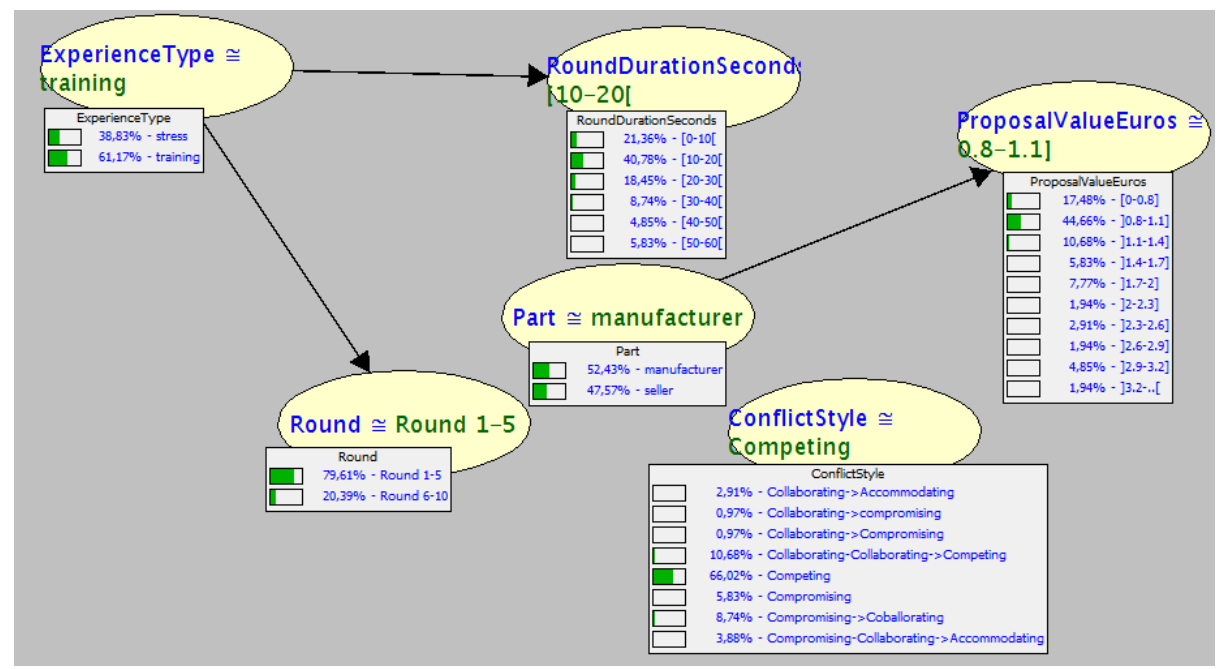

Fig. 4. Representation of the learned network visualized in the SamIam software tool.

probability distribution of the RoundDuration, but if ExperienceType is instantiated, instantiating Round will not change the probability distribution of RoundDuration. This was within the expected outcome.

These results point to the necessity of continuing to develop the experiment in order to gather more data that may uncover the interactions between the variables under analysis.

\section{Conclusions and Future Work}

After performing the experiments and the data analysis described so far, we are able to conclude that it is effectively difficult to extract consistent and reliable behavioural patterns from the collected data. Indeed, when applying machine learning algorithms to the results of the experiments it was noticed that the gathered data was insufficient for a conclusive answer, despite the fact that the raw statistical data was truly interesting.

In order to provide personalized and adapted services to help the conflict resolution field, the need for knowing the most frequent behaviour patterns is very clear. The patterns expressed by users, namely in negotiation processes, may provide vital information for mediation purposes and thus may help to achieve better outcomes. The ability to learn patterns of behaviour became an essential aspect for the successful implementation of IEs. To accomplish this task further developments will include new sources of information that allow more accurate data and the conception of more informative experiments. 


\section{Acknowledgements}

This work is funded by National Funds through the FCT - Fundação para a Ciência e a Tecnologia (Portuguese Foundation for Science and Technology) within projects PEstOE/EEI/UI0752/2011 and PTDC/EEI-SII/1386/2012. The work of Davide Carneiro is also supported by a doctoral grant by FCT (SFRH/BD/64890/2009). The work of Tiago Oliveira is supported by a doctoral grant by FCT (SFRH/BD/85291/2012).

\section{References}

1. Bettini, C., Brdiczka, O., Henricksen, K., Indulska, J., Nicklas, D., Ranganathan, A., Riboni, D.: A survey of context modelling and reasoning techniques. Pervasive and Mobile Computing 6(2), $161-180(2010)$

2. Carneiro, D., Castillo, J.C., Novais, P., Fernndez-Caballero, A., Neves, J.: Multimodal behavioral analysis for non-invasive stress detection. Expert Systems with Applications 39(18), $13376 \quad$ - $13389 \quad$ (2012), http://www.sciencedirect.com/science/article/pii/S0957417412007816

3. Carneiro, D., Gomes, M., Novais, P., Andrade, F., Neves, J.: Automatic classification of personal conflict styles in conflict resolution. In: Atkinson, K.M. (ed.) Legal Knowledge and Information Systems - JURIX 2011: The Twenty-Fourth Annual Conference. pp. 43-52. IOS Press (2011)

4. Carneiro, D., Gomes, M., Novais, P., Neves, J.: Developing dynamic conflict resolution models based on the interpretation of personal conflict styles. In: Antunes, L., Pinto, H. (eds.) Progress in Artificial Intelligence, Lecture Notes in Computer Science, vol. 7026, pp. 44-58. Springer Berlin / Heidelberg (2011)

5. Castillo, J.C., Rivas-Casado, A., Fernández-Caballero, A., López, M.T., Martínez-Tomás, R.: A multisensory monitoring and interpretation framework based on the model-view-controller paradigm. In: Proceedings of the 4th international conference on Interplay between natural and artificial computation - Volume Part I. pp. 441-450. IWINAC'11, Springer-Verlag, Berlin, Heidelberg (2011), http://dl.acm.org/citation.cfm?id=2009405.2009451

6. Cooper, J.O., Heron, T.E., Heward, W.L.: Applied Behavior Analysis. Prentice Hall (1987), http://www.amazon.com/Applied-Behavior-Analysis-John-Cooper/dp/067520223X

7. Fernndez-Caballero, A., Castillo, J.C., Martnez-Cantos, J., Martnez-Toms, R.: Optical flow or image subtraction in human detection from infrared camera on mobile robot. Robotics and Autonomous Systems 58(12), 1273 - 1281 (2010), http://www.sciencedirect.com/science/article/pii/S0921889010001168, ¡ce:title ¿Intelligent Robotics and Neuroscience;/ce:title $i$

8. Gomes, M., Carneiro, D., Novais, P., Neves, J.: Modelling stress recognition in conflict resolution scenarios. In: HAIS (1)'12. pp. 533-544 (2012)

9. Jensen, F.V.: Bayesian networks. Wiley Interdisciplinary Reviews: Computational Statistics 1(3), 307-315 (2009), http://dx.doi.org/10.1002/wics.48

10. Raiffa, H.: The art and science of negotiation: how to resolve conflicts and get the best out of bargaining. The Belknap Press of Harvard University Press (1982)

11. Sheridan, F.: A survey of techniques for inference under uncertainty. Artificial Intelligence Review 5(1), 89-119 (1991)

12. Ye, J., Coyle, L., Dobson, S., Nixon, P.: Using situation lattices to model and reason about context. In: Proceedings of MRC 2007: the Workshop of Modeling and Reasoning Context (coexist with CONTEXT07). pp. 1-12. Roskilde, Denmark (August 2007) 